\title{
Usefulness of a Novel Ultrasonographic Classification Based on Anechoic Area Patterns for Differentiating Warthin Tumors from Pleomorphic Adenomas of the Parotid Gland
}

\author{
Eriko Matsuda,* Takahiro Fukuhara,* Ryohei Donishi,* Katsuyuki Kawamoto,* Yasuaki Hirooka $†$ and Hiromi \\ Takeuchi* \\ *Division of Otolaryngology, Head and Neck Surgery, Department of Sensory and Motor Organs, School of Medicine, Tottori University \\ Faculty of Medicine, Yonago 683-8503, Japan and †Department of Pathobiological Science and Technology, School of Health Science, \\ Tottori University Faculty of Medicine, Yonago 683-8503, Japan
}

\begin{abstract}
Background Ultrasonographic homogeneity is an important differential finding between Warthin tumor and pleomorphic adenoma, two types of benign parotid gland tumors, with the former likely to be heterogeneous and the latter homogeneous. However, differences in the performance of ultrasound machines or the homogeneity cut-off level affect the judgment of ultrasonographic homogeneity. Therefore, in this study, we adopted a novel system for classifying the composition of tumors via ultrasonography, using anechoic area as a substitute for differences in homogeneity to differentiate between Warthin tumors and pleomorphic adenomas.
\end{abstract}

Methods We evaluated 68 tumors that were histopathologically diagnosed as Warthin tumor or pleomorphic adenoma between July 2009 and November 2015. Ultrasonographic images of the tumors were evaluated on the basis of key differentiating features, including features on B-mode imaging and color Doppler imaging. Additionally, the tumors were classified into four groups based on anechoic area, and findings were compared between Warthin tumors and pleomorphic adenomas.

Results While 38 of the tumors were pleomorphic adenomas, 30 were Warthin tumors. The sensitivity, specificity, positive predictive value, negative predictive value, and diagnostic accuracy for detection of Warthin tumors using our novel classification system were $73.3 \%$, $76.3 \%, 71.0 \%, 78.4 \%$ and $75.0 \%$, respectively. Compared to pleomorphic adenomas, Warthin tumors showed large or sponge-like anechoic areas, rich vascularization and an oval shape even at large tumor sizes, and the difference was significant. On defining Warthin tumor as a tumor demonstrating two or more of the findings noted above, the sensitivity, specificity, positive predictive value, negative predictive value and diagnostic accuracy

\footnotetext{
Corresponding author: Eriko Matsuda

e-matsuda@med.tottori-u.ac.jp

Received 2017 September 21

Accepted 2017 November 8

Abbreviation: US, ultrasonography
}

for its detection were $73.3 \%, 84.2 \%, 78.6 \%, 80.0 \%$ and $79.4 \%$, respectively.

Conclusion Our novel classification system based on anechoic area patterns demonstrated by the tumors had high sensitivity, specificity and diagnostic accuracy for differentiating Warthin tumors from pleomorphic adenomas.

Key words diagnostic imaging; parotid neoplasms; pleomorphic adenoma; ultrasonography; Warthin tumor

Approximately $80-90 \%$ of parotid gland tumors are benign, and the majority of these (80-95\%) are pleomorphic adenomas or Warthin tumors, ${ }^{1-3}$ which require different treatment strategies. Malignant transformation occurs in $2-25 \%$ of pleomorphic adenomas, ${ }^{4,5}$ and surgical removal is the preferable treatment for these tumors. In contrast, Warthin tumors can often be left untreated because malignant transformation is extremely rare. Therefore, it is important to differentiate between pleomorphic adenomas and Warthin tumors at the preoperative stage.

Ultrasonography (US) is more advantageous than computed tomography or magnetic resonance imaging because of its simplicity, low cost, non-invasiveness, and real-time images. Therefore, US is the first-line diagnostic imaging method for assessing parotid gland diseases. ${ }^{6}$ It would be ideal if it were possible to differentiate between parotid gland tumors using US and decide if surgery were indicated. Over the last 20 years, many studies have therefore evaluated the usefulness of US in differentiating between pleomorphic adenomas and Warthin tumors of the parotid gland on the basis of shape, homogeneity, anechoic areas, and vascularization. ${ }^{7-15}$

Homogeneity is an important differential finding between the two types of tumor, with Warthin tumors likely to be heterogeneous and pleomorphic adenomas homogeneous. However, a wide range (20-91.9\%) of 
the proportion of homogeneous pleomorphic adenomas has been reported. ${ }^{7-14}$ It has been postulated that this is due to differences in the performance of ultrasound machines or in the homogeneity cut-off level.

Therefore, in this study, we adopted a novel system for classifying the composition of tumors via US, using anechoic area patterns as a substitute for differences in homogeneity; we then evaluated the usefulness of this system for differentiating between Warthin tumors and pleomorphic adenomas.

\section{SUBJECTS AND METHODS Patients}

This was a retrospective study of consecutive patients who underwent US at the Department of Otorhinolaryngology-Head and Neck Surgery of Tottori University Hospital between July 2009 and November 2015, and whose histological diagnosis after resection of parotid gland tumors was either Warthin tumor or pleomorphic adenoma. Patients who did not undergo surgical resection were excluded; this is because parotid tumors have a variety of histopathological presentations, and sufficient diagnostic accuracy cannot be ensured using only cytology. We also excluded one patient who suffered a recurrence and three patients whose preoperative US findings were inconclusive - these included patients with tumors either so large that they could not be fully visualized via US or that were located in the deep lobe of the parotid gland and were therefore difficult to visualize (Fig. 1).

The study was approved by the Ethics Review Board of the Tottori University Faculty of Medicine (1510A064), and requirement for informed consent was waived because of the retrospective nature of the study. All procedures were carry out in accordance with the Declaration of Helsinki of 1975, as revised in 2013.

\section{Methods}

The US images of the Warthin tumors and pleomorphic adenomas were compared and US findings characteristic of each type of tumor were examined.

\section{Assessment of the US images}

US examinations were performed using a Xario XG Ultrasound System (Toshiba Medical Systems Corporation, Tochigi, Japan) until late October 2011 and an ACUSON S2000 Ultrasound System (Siemens Medical Solutions USA, Malvern, PA) from November 2011 onwards. The probe frequency range was $4-9 \mathrm{MHz}$. The images were assessed separately by a board-certified fellow of the Japan Society of Ultrasonics in Medicine (JSUM) and a JSUM registered medical sonographer, both of whom were blinded to histological findings and diagnoses of the tumors. If there were discrepancies between the findings, a final decision was reached by consensus.

\section{B-mode imaging}

Images of the tumors were evaluated on the basis of the

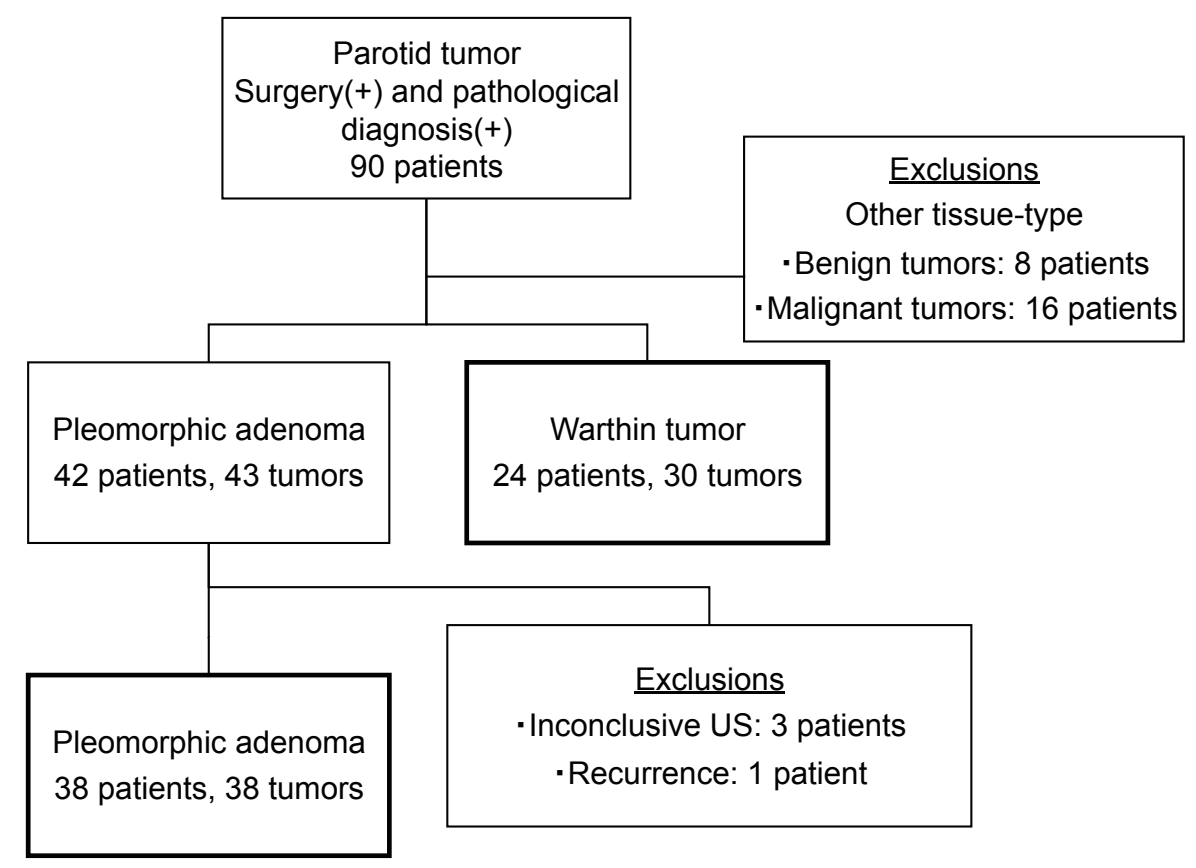

Fig. 1. Flowchart of inclusion and exclusion criteria. US, ultrasonography. 


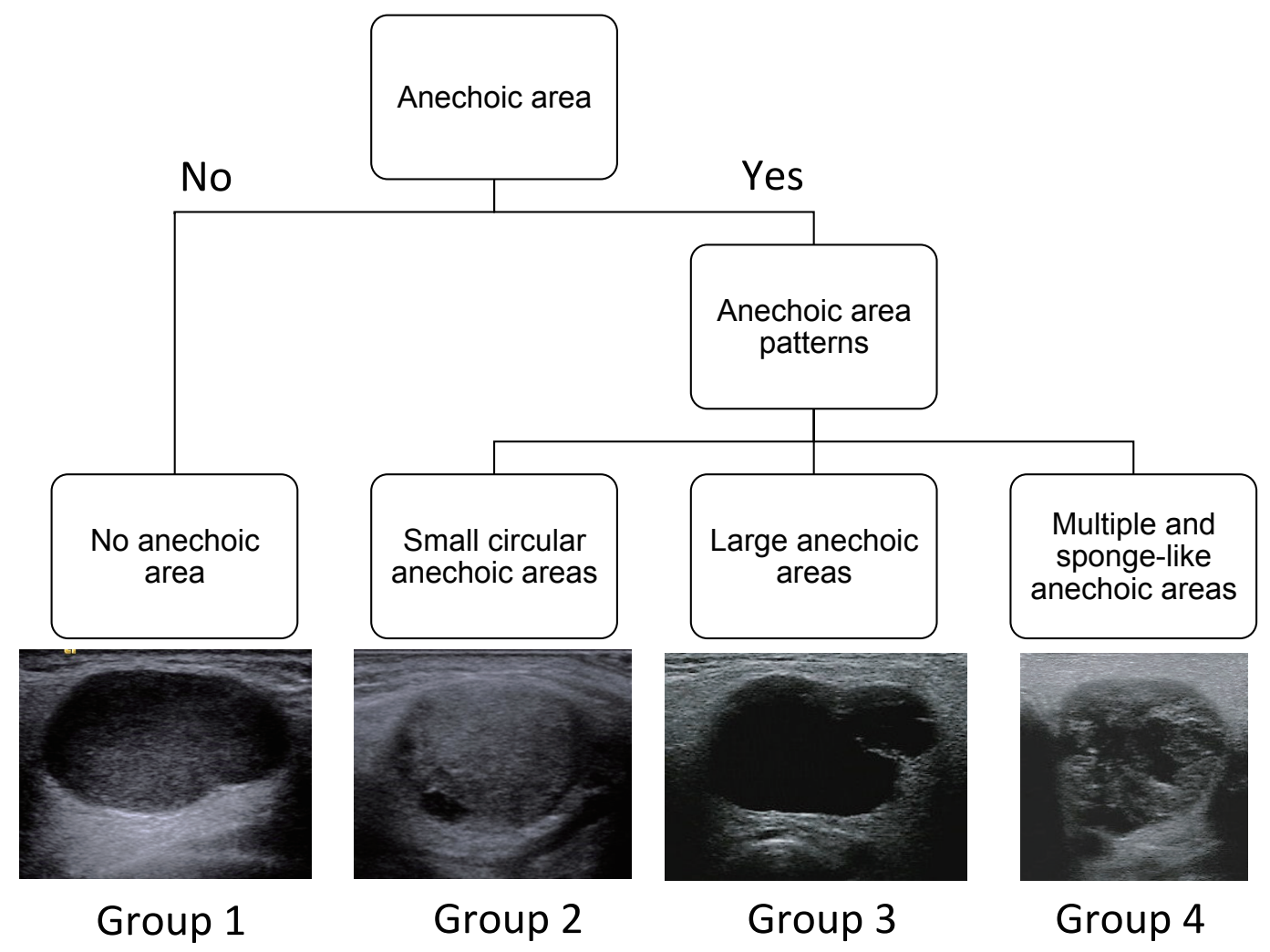

Fig. 2. Classification based on anechoic area patterns.

following parameters: major axis, shape (oval or lobulated/irregular), borders (well-defined or ill-defined), internal echo levels (hypoechoic, isoechoic or hyperechoic compared with normal parts of the gland) and presence or absence of posterior echo enhancement.

In addition, the tumors were classified into four groups on the basis of anechoic area patterns on US: Tumors with no anechoic areas were allocated to Group 1; tumors with one or a few small circular anechoic areas $(<3 \mathrm{~mm})$ were allocated to Group 2; tumors with large anechoic areas and no sponge-like appearance were allocated to Group 3; and tumors with a sponge-like appearance, in which large and small anechoic areas were mixed finely within the solid part, were allocated to Group 4 (Fig. 2).

\section{Doppler imaging}

Vascularization of the tumors was evaluated according to three grades on the basis of the amount of flow signals on color Doppler imaging. Thus, tumors with no flow signal or with one or two punctiform flow signals were classified as Grade 1; those with three to five punctiform or linear flow signals in or around the tumor were classified as Grade 2; and those with more than five flow signals in the entire area of the solid part of the tumor were classified as Grade 3.

\section{Statistical analysis}

Non-normally distributed continuous data were expressed as median [range], and categorical data as $\mathrm{n}$ (\%). SPSS Statistics for windows, version 22.0 (IBM, Armonk, NY) was used for all statistical analyses. For continuous variables, the Mann-Whitney $U$ test was used to compare differences between the two groups, and all continuous variables were expressed as the median and range. The Chi-Square test or Fisher's exact test was used to compare categorical variables, and all categorical variables were expressed as numbers and percentages. A $P$-value $<0.05$ was considered statistically significant.

Table 1. Patient data

\begin{tabular}{lll}
\hline & Warthin tumor & Pleomorphic adenoma \\
\hline Patients $(n)$ & 24 & 38 \\
Age (years) & $55(46-80)$ & $65(28-80)$ \\
Sex (men/women) & $19 / 5$ & $12 / 26$ \\
\hline
\end{tabular}

Median [range]. 
Table 2. Gray-scale ultrasonographic (US) features of Warthin tumors and pleomorphic adenomas of the parotid gland

\begin{tabular}{lccc}
\hline US features & Warthin tumor & Pleomorphic adenoma & $P$-values \\
\hline Tumors $(n)$ & 30 & 38 & $0.008^{* *}$ \\
Diameter $(\mathrm{mm})$ & $30.5(16.7-46.3)$ & $22.4(9.3-52.0)$ & $0.012^{*}$ \\
Shape & $21(70.0 \%)$ & $15(39.5 \%)$ & 1.000 \\
$\quad$ Oval & $9(30.0 \%)$ & $23(60.5 \%)$ & 1.000 \\
$\quad$ Lobulated/irregular & $30(100 \%)$ & $37(97.4 \%)$ & \\
Border & $0(0 \%)$ & $1(2.6 \%)$ & \\
$\quad$ Well-defined & $29(96.7 \%)$ & $37(97.4 \%)$ & $1(2.6 \%)$ \\
$\quad$ Ill-defined & $1(3.3 \%)$ & $0(0 \%)$ & 0.500 \\
Internal echo level & $0(0 \%)$ & $36(94.7 \%)$ & \\
$\quad$ Hypoechoic & & $2(5.3 \%)$ & \\
Isoechoic & $30(100 \%)$ & & \\
Pyperechoic & $0(0 \%)$ & & \\
$\quad$ Yes & & & \\
$\quad$ No & & & \\
\hline
\end{tabular}

Median [range]; $* P<0.05 ; * * P<0.01$.

\begin{tabular}{|c|c|c|c|}
\hline US feature & Warthin tumor & Pleomorphic adenoma & $P$-value \\
\hline Anechoic areas & & & $<0.001 * * *$ \\
\hline Group 1 & $6(20.0 \%)$ & $24(63.2 \%)$ & \\
\hline Group 2 & $2(6.7 \%)$ & $5(13.2 \%)$ & \\
\hline Group 3 & $6(20.0 \%)$ & $7(18.4 \%)$ & \\
\hline Group 4 & $16(53.3 \%)$ & $2(5.3 \%)$ & \\
\hline
\end{tabular}
Table 4. Doppler-mode ultrasonographic (US) features of Warthin tu-
mors and pleomorphic adenomas of the parotid gland

\begin{tabular}{cccc}
\hline US feature & Warthin tumor & Pleomorphic adenoma & $P$-value \\
\hline Vascularization & & & $<0.001^{* * *}$ \\
Grade 1 & $10(33.3 \%)$ & $30(78.9 \%)$ & \\
Grade 2 & $7(23.3 \%)$ & $5(13.2 \%)$ & \\
Grade 3 & $13(43.3 \%)$ & $3(7.9 \%)$ & \\
\hline$* * * P<0.001$ & & &
\end{tabular}

A total of 68 tumors were included in the study; 30 Warthin tumors and 38 pleomorphic adenomas. The findings from the B-mode images are shown in Table 2.

The median value of the major axis was $30.5 \mathrm{~mm}$ (range, 16.7 to $46.3 \mathrm{~mm}$ ) for Warthin tumors and $22.4 \mathrm{~mm}$ (range, 9.3 to $52.0 \mathrm{~mm}$ ) for pleomorphic adenomas $(P=0.008)$. Morphologically, $70.0 \%$ of Warthin tumors were oval, whereas $60.5 \%$ of pleomorphic adenomas were lobulated $(P=0.012)$. Assuming that an oval shape was characteristic of Warthin tumor, the sensitivity, specificity, and diagnostic accuracy for detection of this tumor were $70.0 \%, 60.5 \%$ and $64.7 \%$, respectively. There was no difference in clarity of the borders, internal echo levels, or posterior echo enhancement between the two types of tumors.

Based on anechoic area patterns, $20.0 \%$ of Warthin tumors and $63.2 \%$ of pleomorphic adenomas were classified as Group 1, 6.7\% of Warthin tumors and $13.2 \%$ of pleomorphic adenomas as Group 2, 20.0\% of Warthin tumors and $18.4 \%$ of pleomorphic adenomas as Group 3, and $53.3 \%$ of Warthin tumors and $5.3 \%$ of pleomorphic adenomas as Group 4; there were significant differences between the groups $(P<0.001)$ (Table 3$)$.

Assuming that tumors in Groups 3 and 4 had features characteristic of Warthin tumors, the sensitivity, specificity, positive predictive value, negative predictive 
Table 5. Diagnostic accuracy of each ultrasonographic (US) feature differentiating Warthin tumors from pleomorphic adenomas

\begin{tabular}{llllll}
\hline US features & Sensitivity & Specificity & PPV & NPV & Accuracy \\
\hline Shape & $70.0 \%$ & $60.5 \%$ & $58.3 \%$ & $71.9 \%$ & $64.7 \%$ \\
Anechoic area pattern & $73.3 \%$ & $76.3 \%$ & $71.0 \%$ & $78.4 \%$ & $75.0 \%$ \\
Vascularization & $66.7 \%$ & $78.9 \%$ & $71.4 \%$ & $75.0 \%$ & $73.5 \%$ \\
Presence of two or more than two features & $73.3 \%$ & $84.2 \%$ & $78.6 \%$ & $80.0 \%$ & $79.4 \%$ \\
\hline
\end{tabular}

NPV, negative predictive value; PPV, positive predictive value.
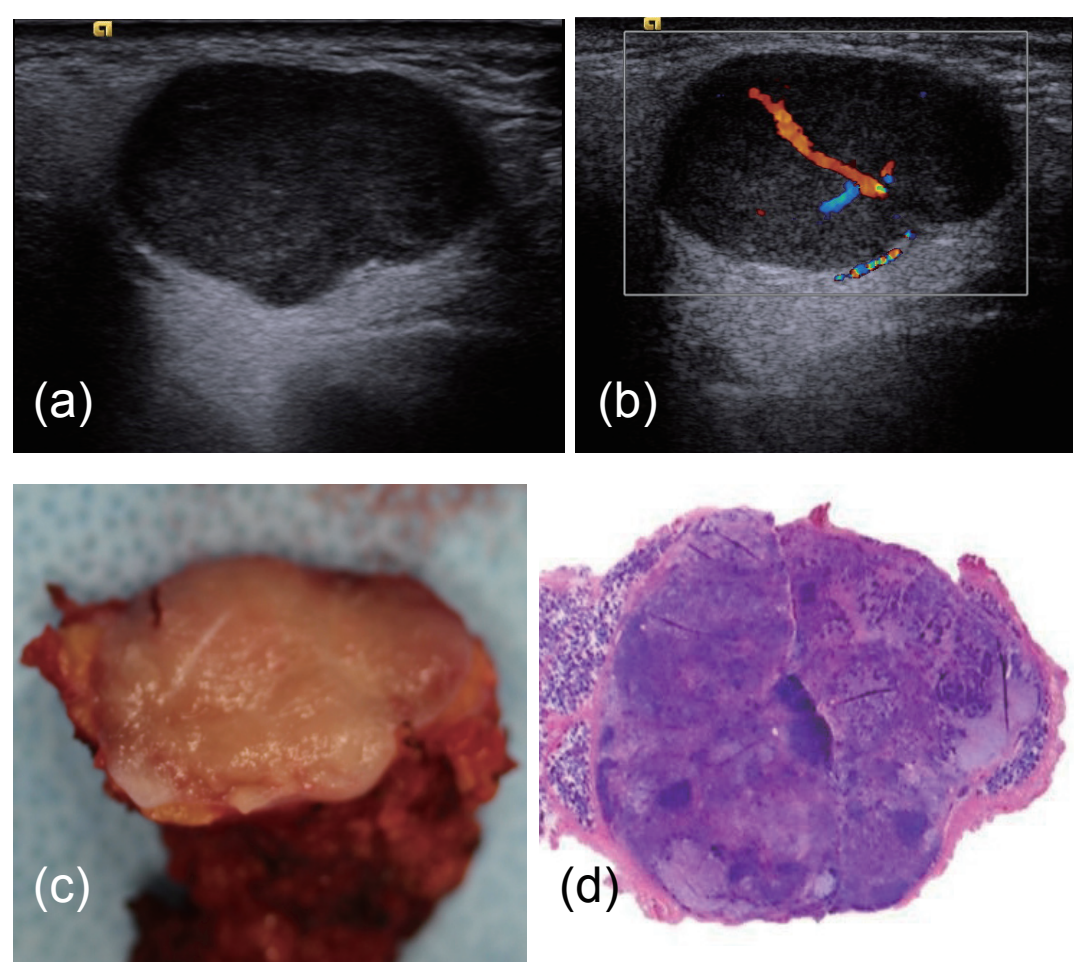

Fig. 3. Ultrasonography images of a pleomorphic adenoma: macroscopic/histological findings of resected specimens. Note the lobulated shape of the tumor, without anechoic area and one linear flow signal (a, b). Macroscopic findings of the resected specimen show a well-defined boundary with the surrounding tissues; the sectioned surface is glossy, and the tumor is solid (c). Histological findings show that the tumor is covered with a fibrous capsule and that the tumor content is solid, but mixed with epithelial components, myxomatous stroma, and cartilaginous matrix-like stroma (d). value, and diagnostic accuracy for detection of Warthin tumor in our study were $73.3 \%, 76.3 \%, 71.0 \%, 78.4 \%$ and $75.0 \%$, respectively.

The results of evaluation of the differences in vascularization were as follows: Grade 1 in $33.3 \%$ and $78.9 \%$ of Warthin tumors and pleomorphic adenomas, respectively; Grade 2 in $23.3 \%$ and $13.2 \%$, respectively; and Grade 3 in $43.3 \%$ and $7.9 \%$, respectively. This indicates that vascularization was significantly more abundant in the Warthin tumors $(P<0.001)$ (Table 4$)$.

Assuming Grade 2 vascularization or above was characteristic of Warthin tumors, the sensitivity, specificity, and diagnostic accuracy for detection of Warthin tumor were $66.7 \%, 78.9 \%$ and $73.5 \%$, respectively.

The most useful ultrasonographic findings for differentiating between Warthin tumors and pleomorphic adenomas were the tumor's shape, anechoic area pattern, and vascularization. (Figs. 3 and 4) When we defined
Warthin tumor as that satisfied two or more of the findings noted above, the sensitivity, specificity, positive predictive value, negative predictive value and diagnostic accuracy for detection of Warthin tumors were 73.3\%, $84.2 \%, 78.6 \%, 80.0 \%$ and $79.4 \%$, respectively (Table 5).

\section{DISCUSSION}

Although the level of homogeneity within parotid tumors has been a useful characteristic for differentiating Warthin tumors from pleomorphic adenomas using US, ${ }^{7}$ the range of reported homogeneity varies between $20 \%$ and $91.9 \%,,^{7-14}$ and the proportion of homogeneous pleomorphic adenomas was higher more than 15 years ago compared to that in recent reports. ${ }^{7}$ This supports the postulation that the performance of ultrasound machine has an effect on assessment of levels of homogeneity of pleomorphic adenomas as new ultrasound machines, which have higher resolution, can enable visualization 

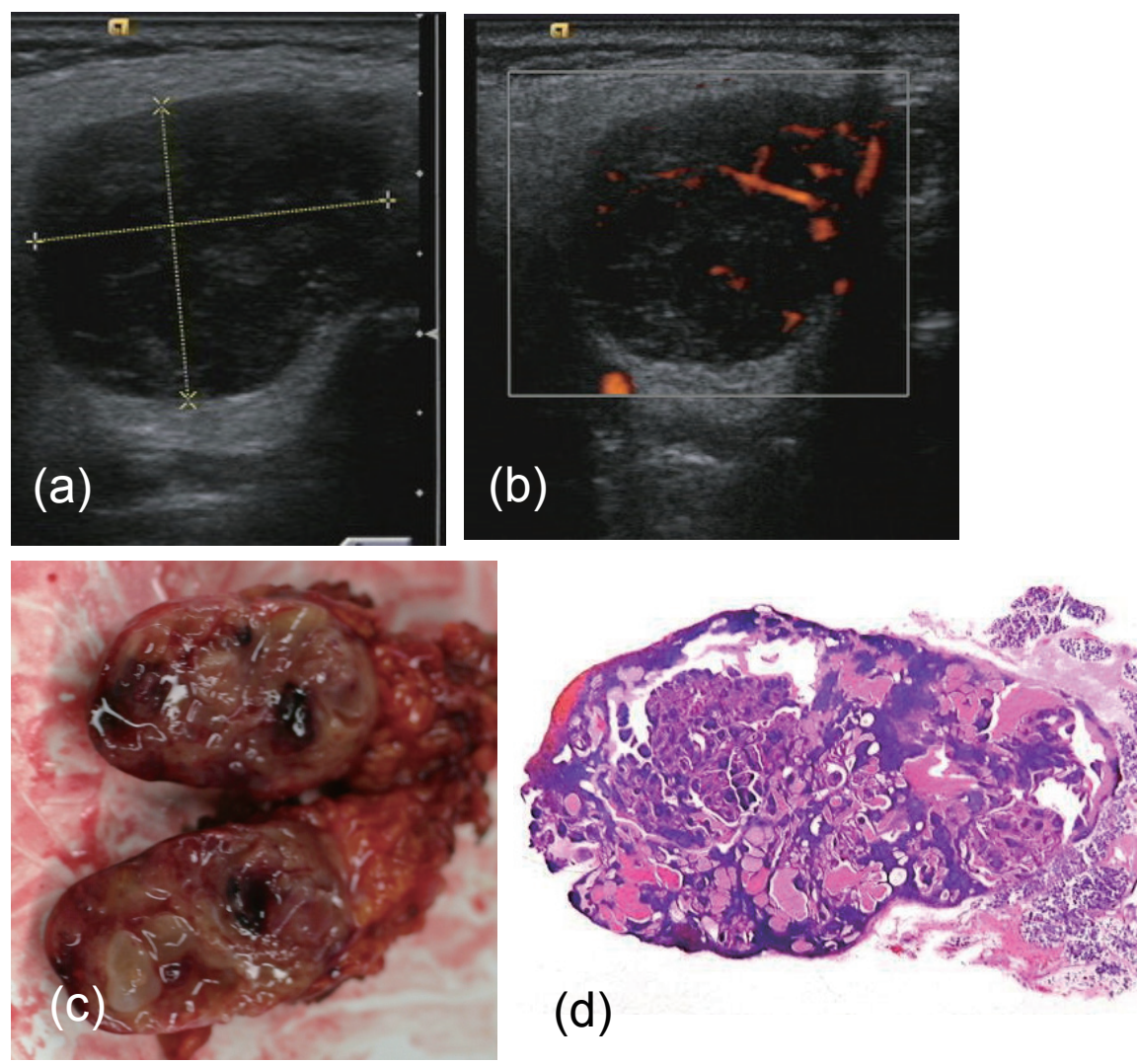

(d)
Fig. 4. Ultrasonography images of a Warthin tumor: macroscopic/histological findings of resected specimens. Note the tumor contains many large and small anechoic areas; additionally, a large number of punctiform and linear flow signals can be seen in the solid part of the tumor (a, b). Macroscopic findings show that the solid and cystic parts of the tumor are mixed together (c). Note that the solid parts of the tumor contain dense epithelial cells and that the cystic parts of the tumor are of various sizes (d). of the pathological heterogeneity of the internal architecture of pleomorphic adenomas. This is thought to be another reason for the wide range of frequencies of homogeneous pleomorphic adenomas among recent studies, as the degree of homogeneity that marks the cut-off level is determined by subjective assessment of the US image. In addition, it is difficult to assess homogeneity of the solid parts via gray-scale US when the tumor has mixed content that includes many small solid parts and spongiform cysts as solid parts may be too small for assessment.

For these reasons, in this study, we assessed the sonographic composition of tumors using anechoic area patterns rather than sonographic homogeneity. We classified $76.3 \%$ of pleomorphic adenomas as Group 1 or 2 because they were characterized by no anechoic areas or a few small circular anechoic areas. Our classification is supported by previous studies that have reported that pleomorphic adenomas are characterized mainly by an absence of anechoic areas or presence of a small anechoic area. ${ }^{9}$ Further, we classified $73.3 \%$ of the Warthin tumors as Group 3 or 4 based on their anechoic area patterns; these tumors were characterized by large or sponge-like anechoic areas. Some studies have reported a high frequency of anechoic areas in Warthin tumors
44.8-93.3\%;7, 8, 10, 11, 14 in one study, for example, $96.6 \%$ of Warthin tumors contained pathologically identified cystic lesions. ${ }^{11}$ However, the diagnostic accuracy using anechoic areas or sponge-like anechoic areas as characteristic features of Warthin tumor has been reported to be $44.4-56.3 \%,{ }^{11}, 14$ which was not high enough. In contrast, our novel classification system based on anechoic area patterns of tumor content has demonstrated high diagnostic accuracy for differentiating Warthin tumors from pleomorphic adenomas.

When we measured the tumors, we found that the diameters of Warthin tumors were significantly larger than those of pleomorphic adenomas. However, this result may be subject to bias because the tumors in this study were from operated patients; surgery often was not indicated for small Warthin tumors, which were therefore excluded from this study, while pleomorphic adenomas were resected even if they were small. For this reason, tumor diameter was not used to differentiate Warthin tumors from pleomorphic adenomas on US.

Pleomorphic adenomas have been found to be more likely to have a lobulated shape when their diameter exceeds approximately $20 \mathrm{~mm} ;{ }^{16}$ in our study, large pleomorphic adenomas also tended to have a lobulated shape. In contrast, we found that Warthin tumors were 
oval shaped even when they had a large size. This finding was similar to those in previous studies. ${ }^{9-11,13}$

We also found that the vascularization within Warthin tumors was significantly richer than that in pleomorphic adenomas. This finding was also similar to those in previous studies. ${ }^{12-14}$ In one study, microvessels were found to be less numerous in pleomorphic adenomas, and Warthin tumors had higher cellularity and microvessel density than pleomorphic adenomas. ${ }^{17}$ However, a few pleomorphic adenomas had rich vascularity in our study. This apparent disparity can be explained by findings from some studies that vascularity is rich in the presence of abundant cellular components but that it decreases in the presence of abundant myxomatous stroma. ${ }^{17}$

There were several limitations of this study. First, this was a retrospective study and thus US features were not evaluated in real time. Second, the sample size was small, with only 68 tumors; further, larger studies are required. Third, this study was limited to pleomorphic adenomas and Warthin tumors of the parotid gland; therefore, other benign and malignant tumors, albeit comparatively rare, were excluded. Fourth, we relied on the accuracy of pathological diagnosis and excluded patients who had not undergone surgery; therefore, approximately half of the patients with suspected Warthin tumors were excluded.

In conclusion, our novel classification system based on anechoic area patterns of the content of tumors had high sensitivity, high specificity and diagnostic accuracy for differentiating Warthin tumors from pleomorphic adenomas. Furthermore, the combination of two or more ultrasonographic findings (relating to anechoic area patterns, shape, and vascularization) may improve diagnostic accuracy when differentiating Warthin tumors from pleomorphic adenomas and has considerable advantages over previous diagnostic systems.

\section{The authors declare no conflict of interest.}

\section{REFERENCES}

1 Guzzo M, Locati LD, Prott FJ, Gatta G, McGurk M, Licitra L. Major and minor salivary gland tumors. Crit Rev Oncol Hematol. 2010;74:134-48. PMID: 19939701.

2 Bradley PJ, McGurk M. Incidence of salivary gland neoplasms in a defined UK population. Br J Oral Maxillofac Surg. 2013;51:399-403. PMID: 23103239.

3 Tian Z, Li L, Wang L, Hu Y, Li J. Salivary gland neoplasms in oral and maxillofacial regions: a 23-year retrospective study of 6982 cases in an eastern Chinese population. Int J Oral Maxillofac Surg. 2010;39:235-42. PMID: 19951834.

4 Renehan A, Gleave EN, McGurk M. An analysis of the treatment of 114 patients with recurrent pleomorphic adenomas of the parotid gland. Am J Surg. 1996;172:710-4. PMID: 8988685.

5 Park GC, Cho KJ, Kang J, Roh JL, Choi SH, Kim SY, et al. Relationship between histopathology of pleomorphic adenoma in the parotid gland and recurrence after superficial parotidectomy. J Surg Oncol. 2012;106:942-6. PMID: 22740329.

6 Petrovan C, Nekula DM, Mocan SL, Voidăzan TS, Coşarcă A. Ultrasonography-histopathology correlation in major salivary glands lesions. Rom J Morphol Embryol. 2015;56:491-7. PMID: 26193218.

7 Shimizu M, Ussmüller J, Hartwein J, Donath K, Kinukawa N. Statistical study for sonographic differential diagnosis of tumorous lesions in the parotid gland. Oral Surg Oral Med Oral Pathol Oral Radiol Endod. 1999;88:226-33. PMID: 10468468.

8 Zajkowski P, Jakubowski W, Białek EJ, Wysocki M, Osmólski A, Serafin-Król M. Pleomorphic adenoma and adenolymphoma in ultrasonography. Eur J Ultrasound. 2000;12:23-9. PMID: 10996767.

9 Bialek EJ, Jakubowski W, Karpinska G. Role of ultrasonography in diagnosis and differentiation of pleomorphic adenomas: work in progress. Arch Otolaryngol Head Neck Surg. 2003;129:929-33. PMID: 12975263.

10 Kim J, Kim EK, Park CS, Choi YS, Kim YH, Choi EC. Characteristic sonographic findings of Warthin's tumor in the parotid gland. J Clin Ultrasound. 2004;32:78-81. PMID: 14750138.

11 Yuan WH, Hsu HC, Chou YH, Hsueh HC, Tseng TK, Tiu CM. Gray-scale and color Doppler ultrasonographic features of pleomorphic adenoma and Warthin's tumor in major salivary glands. Clin Imaging. 2009;33:348-53. PMID: 19712813.

12 Knopf A, Mansour N, Chaker A, Bas M, Stock K. Multimodal ultrasonographic characterisation of parotid gland lesions--a pilot study. Eur J Radiol. 2012;81:3300-5. PMID: 22269165.

13 Rong X, Zhu Q, Ji H, Li J, Huang H. Differentiation of pleomorphic adenoma and Warthin's tumor of the parotid gland: ultrasonographic features. Acta Radiol. 2014;55:1203-9. PMID: 24324278.

14 Miao LY, Xue H, Ge HY, Wang JR, Jia JW, Cui LG. Differentiation of pleomorphic adenoma and Warthin's tumour of the salivary gland: is long-to-short diameter ratio a useful parameter? Clin Radiol. 2015;70:1212-19. PMID: 26216455.

15 Martinoli C, Derchi LE, Solbiati L, Rizzatto G, Silvestri E, Giannoni M. Color Doppler sonography of salivary glands. AJR Am J Roentgenol. 1994;163:933-41. PMID: 8092039.

16 Japan Association of Breast and Thyroid Sonology. Thyroid Ultrasound-A guidebook for diagnosis and management, 2nd Edition. Tokyo, Japan: Nankodo. 2012.

17 Yabuuchi H, Fukuya T, Tajima T, Hachitanda Y, Tomita K, Koga M. Salivary gland tumors: diagnostic value of gadolinium-enhanced dynamic MR imaging with histopathologic correlation. Radiology. 2003;226:345-54. PMID: 12563124. 